\title{
The Role of Sleep Problems in Central Pain Processing in Rheumatoid Arthritis
}

\author{
Yvonne C. Lee, ${ }^{1}$ Bing Lu,${ }^{1}$ Robert R. Edwards, ${ }^{1}$ Ajay D. Wasan, ${ }^{1}$ Nicholas J. Nassikas, ${ }^{1}$ \\ Daniel J. Clauw, ${ }^{2}$ Daniel H. Solomon, ${ }^{1}$ and Elizabeth W. Karlson ${ }^{1}$
}

\begin{abstract}
Objective. Among rheumatoid arthritis (RA) patients, the intensity of pain may be out of proportion to the severity of peripheral inflammation. This observation suggests that mechanisms of central nervous system pain amplification, such as diminished conditioned pain modulation (CPM), may play a role in enhancing pain perception among some RA patients. This study was undertaken to examine the level of CPM, pressurepain threshold, and pressure-pain tolerance among RA patients compared to healthy controls.
\end{abstract}

Methods. Fifty-eight female RA patients and 54

The content of this article is solely the responsibility of the authors and does not necessarily represent the official views of Harvard Catalyst, Harvard University and its affiliated academic health care centers, or the NIH.

Supported by Harvard Catalyst | The Harvard Clinical and Translational Science Center, which is funded through the National Center for Research Resources and the National Center for Advancing Translational Sciences (NIH grant UL1-RR-025758) and by financial contributions from Harvard University and its affiliated academic health care centers. Dr. Lee's work was supported by the NIH (grant AR-057578). Dr. Edwards' work was supported by the NIH (grants AG-034982 and AR-057920). Dr. Solomon received salary support from the NIH (grant AR-055989). Dr. Karlson's work was supported by the NIH (grants AR-49880, AR-047782, and AR-052403).

${ }^{1}$ Yvonne C. Lee, MD, MMSc, Bing Lu, MD, DrPH, Robert R. Edwards, PhD, Ajay D. Wasan, MD, MSc, Nicholas J. Nassikas, BA, Daniel H. Solomon, MD, MPH, Elizabeth W. Karlson, MD: Brigham and Women's Hospital, Boston, Massachusetts; ${ }^{2}$ Daniel J. Clauw, MD: University of Michigan, Ann Arbor.

Dr. Lee owns stock or stock options in Merck, Novartis, and Elan and has received research grants from Forest Research Institute. Dr. Clauw has received consulting fees from Jazz Pharmaceuticals, Pfizer, Eli Lilly, Forest Laboratories, Pierre Fabre Pharmaceuticals, UCB, Nuvo Research, and Merck \& Company (less than $\$ 10,000$ each) and has received research grants from Pfizer, Nuvo Research, and Forest Laboratories. Dr. Solomon has received research grants from Amgen, Abbott, and Eli Lilly and has served as an uncompensated member of committees overseeing pain medication clinical trials sponsored by Pfizer.

Address correspondence to Yvonne C. Lee, MD, MMSc, Division of Rheumatology, Immunology, and Allergy, Brigham and Women's Hospital, 75 Francis Street, PBB-B3, Boston, MA 02115. E-mail: ylee9@partners.org.

Submitted for publication March 26, 2012; accepted in revised form September 27, 2012. age-matched female control subjects without chronic pain underwent quantitative sensory testing (QST) to assess CPM levels, pressure-pain thresholds, and pressure-pain tolerance levels. CPM was induced using a cold water bath, and the pain threshold (when patients first felt pain) and pain tolerance (when pain was too much to bear) were assessed with an algometer. Associations between RA and each QST outcome were analyzed using linear regression. Sleep problems, mental health, and inflammation were assessed as mediators of the relationship between RA and QST outcomes.

Results. The median CPM level was $0.5 \mathrm{~kg} / \mathrm{cm}^{2}$ (interquartile range $[I Q R]-0.1,1.6)$ among RA patients, compared to a median of $1.5 \mathrm{~kg} / \mathrm{cm}^{2}$ (IQR -0.1 , $2.5)$ among controls $(P=0.04)$. RA patients, compared to controls, had a lower pain threshold and lower pain tolerance at the wrists (each $P \leq \mathbf{0 . 0 5}$ ). In addition, RA patients had greater problems with sleep, pain catastrophizing, depression, and anxiety $(P<0.0001$ versus controls). Results of mediation analyses suggested that low CPM levels might be attributed, in part, to sleep disturbance $(P=0.04)$.

Conclusion. RA patients have impaired CPM when compared to pain-free control subjects. Sleep problems may mediate the association between RA and attenuated CPM.

Pain is the most common and disabling symptom of rheumatoid arthritis (RA). Although physicians often assume that inflammation is the stimulus for pain, many RA patients continue to have pain despite having adequately suppressed inflammation (1). Studies of experimental pain sensitivity have shown that the pressurepain threshold (i.e., the degree of pressure that first elicits pain) is lower in RA patients than in healthy controls $(2,3)$. These thresholds are lower in the joints as well as at sites outside the joints $(4,5)$. The widespread distribution of hyperalgesia suggests that the underlying 
mechanisms of pain originate from the central nervous system (CNS), rather than at focal sites of peripheral inflammation.

In animal models, one well-described CNS mechanism for modulating pain involves descending analgesic pathways, being those that "descend" from the cerebral cortex, hypothalamus, and brainstem to regulate peripheral sensory input in the spinal cord (6). In response to acutely painful stimuli, inhibitory pathways are activated, leading to a diffuse decrease in pain. In humans, the effects of these pathways may be seen when the perception of noxious stimuli, such as arthritis-related pain, is momentarily attenuated after experiencing another strong noxious stimulus, such as stubbing one's toe. In the experimental setting, this phenomenon has been termed "loss of diffuse noxious inhibitory controls," or diminished conditioned pain modulation (CPM) (7). The level of CPM can be measured with a noninvasive test and is a sensitive measure of deficits in central pain modulation in chronic, widespread pain conditions (8).

In this study, we measured the level of CPM, the pressure-pain threshold, and the pressure-pain tolerance (i.e., the degree of pressure that elicits unbearable pain) among RA patients and age-matched healthy control subjects. We also assessed the role of sleep problems, mental health, and markers of inflammation in mediating differences in CPM levels and pain sensitivity. Variables were considered mediators if, when added to the models, they partially or completely diminished the association between RA and the outcomes of CPM, pressure-pain threshold, or pressure-pain tolerance. We hypothesized that RA patients would have diminished CPM, a lower pressure-pain threshold, and a lower pressure-pain tolerance when compared with these same sensory measures in controls. We also hypothesized that sleep problems and greater pain catastrophizing would mediate the differences in CPM levels, pressure-pain thresholds, and pain tolerances between RA patients and controls.

\section{PATIENTS AND METHODS}

Study population. This study compared quantitative sensory testing (QST) measures, including measurements of the CPM analgesic response, the pressure-pain threshold, and the pressure-pain tolerance, between RA patients and agematched healthy control subjects. We recruited female RA patients from the rheumatology clinics of an academic medical center, and identified age-matched female control subjects from a hospital-based registry for study volunteers and from the surrounding community between April 2010 and July 2011. Inclusion criteria included female sex and age $\geq 40$ years. We limited the study to women, because RA occurs predominantly in women and because women are known to have lower pain threshold and pain tolerance compared to men. Exclusion criteria included the use of opioid pain medications and the presence of cold-sensitive conditions. We required that the diagnosis of RA in patients be confirmed by a board-certified rheumatologist.

Control subjects were recruited to match RA patients by age. Exclusion criteria for controls included 1) history of a systemic inflammatory disease (e.g., RA, systemic lupus erythematosus, seronegative spondylarthropathy), 2) history of a chronic pain condition (e.g., osteoarthritis, fibromyalgia, migraine headaches, irritable bowel syndrome), and 3) chronic pain for any other reason. The Institutional Review Board of Partners Healthcare approved the study. All participants provided their written informed consent.

Assessment of clinical variables. A board-certified rheumatologist (VCL) performed a physical examination of each subject, including determination of the tender and swollen joint counts. Blood samples were collected to assess the serum levels of C-reactive protein (CRP), tumor necrosis factor $\alpha$ (TNF $\alpha$ ), and interleukin-6 (IL-6). The extent of disease activity in RA patients was determined using the Disease Activity Score in 28 joints (DAS28), calculated from the tender joint count, swollen joint count, and CRP level (9). Participants with a DAS28 $<2.6$ are considered in remission, whereas a DAS28 between 2.6 and 3.2 is considered low disease activity, and a DAS28 $>5.1$ indicates high disease activity. In addition, a list of all current medications was obtained from each subject.

Clinical pain severity was assessed using the current pain numeric rating scale (0-10) on the Brief Pain InventoryShort Form, a 9-question survey regarding the sensory and reactive aspects of clinical pain. The subjects' mental health was measured using the Hospital Anxiety and Depression Scale (HADS), a validated 14-item questionnaire that assesses depression and anxiety in physically ill patients (10). A score $\geq 8$ on the anxiety or depression subscales suggests difficulties with anxiety or depression. We quantified sleep disturbances using the Medical Outcomes Study (MOS) Sleep Scale (Sleep Problems Index II), a validated 12-item questionnaire that assesses sleep problems in chronically ill patients (11). A score $>35$ indicates sleep problems. The extent of pain catastrophizing was measured using the Pain Catastrophizing Scale, a validated 13-item scale that assesses a set of negative cognitive and emotional processes, including helplessness, pessimism, rumination, and magnification of symptoms (12). Scores on this scale range $0-52$ (higher scores indicate greater catastrophizing). All data were double-entered by 2 individuals, and entries were checked against each other for accuracy.

Quantitative sensory testing. QST techniques consisted of measurement of the pressure-pain threshold and pressure-pain tolerance levels, as well as a paradigm to test CPM. One rheumatologist (YCL), trained in QST, performed all testing. She explained the procedures using standard scripts and was blinded to each subject's case or control status. To ensure that a subject's RA status, as would be evident on physical examination, was masked, each participant wore large cotton gloves to cover the hands and wrists, the most common sites of RA-related inflammation and structural damage.

All pain threshold and pain tolerance tests were performed twice on the same day, with 2-5 minutes separating 
each test. The first test was designated a trial run, to accustom participants to the testing procedures. The second test was designated the test run, from which all data were obtained. Tests were performed on the same day to minimize heterogeneity caused by daily changes in environment, disease activity, and mental status. Our previous studies have indicated that pressure-pain thresholds and tolerances are highly reproducible when testing is done on the same day (13).

Pressure-pain threshold and pressure-pain tolerance testing was performed on the joints (at the dorsal wrists, on the ulnar side of the extensor pollicis longus tendon, and at the knees, immediately proximal to the patella), at sites close to the joints (the thumbnails), and at sites distant from the joints (superior trapezius muscles), using a Wagner Instruments FPK 20 algometer. This instrument has an accuracy of \pm 2 graduations for capacities up to 2,500 grams, and \pm 1 graduation for capacities larger than 2,500 grams. It provides reproducible measurements of pain threshold and pain tolerance among RA patients, with intraclass correlations ranging from 0.75 to 0.92 (13).

Pressure was increased at a rate of $\sim 1 \mathrm{~kg} / \mathrm{second}$ to a maximum of $11 \mathrm{~kg}$. The pressure-pain threshold was defined as the degree of pressure when the participants first felt pain. The pressure-pain tolerance level was defined as the degree of pressure when the participants wanted to stop testing due to a sensation of "too much pain." The order of testing was as follows: 1) the thumbnails, 2) the wrists, 3) the trapezius muscles, and 4) the knees. The order was standardized to ensure uniformity of study measurements. The order was chosen such that areas outside the joints were interspersed with joint-specific areas, minimizing the risk of systematic bias toward the joints, relative to nonjoint sites. All assessments were performed bilaterally.

The CPM paradigm was performed using a conditioning stimulus (a relatively tonic noxious stimulus that leads to activation of CPM) and a test stimulus (a briefly painful stimulus used to evaluate the analgesic response to the conditioning stimulus) (14-16). The conditioning stimulus was immersion of the right hand in a cold water bath at $6^{\circ} \mathrm{C}$, and the test stimulus was application of pressure to the left superior portion of the trapezius muscle. Participants were instructed to put their hand in the water bath for 30 seconds. The pressurepain threshold was assessed immediately before immersion and 20 seconds after immersion, while each participant's hand remained in the cold water. The magnitude of CPM was defined as the change in pressure-pain threshold from baseline to 20 seconds after cold water immersion. Positive scores are indicative of a greater analgesic response to the cold pressor test (i.e., higher CPM levels).

Power calculation. The standard deviation of CPM among RA patients and healthy control subjects was estimated from a small study of 21 RA patients and 21 healthy controls (4). With an alpha level of 0.05 and a standard deviation of $1.5 \mathrm{~kg} / \mathrm{cm}^{2}$, a sample size of $50 \mathrm{RA}$ patients and 50 controls would provide $90 \%$ power to detect a difference in the CPM of $1.0 \mathrm{~kg} / \mathrm{cm}^{2}$. Additional participants were enrolled to ensure adequate power, in case the data were not obtainable from all participants. This precaution was undertaken to account for participants with pressure-pain thresholds exceeding the upper limit of detection $\left(11 \mathrm{~kg} / \mathrm{cm}^{2}\right)$ at baseline, because further increases in the pressure-pain threshold would not be detectable.

Statistical analysis. All statistical analyses were performed using the SAS statistical software package (version 9.2). Results for continuous variables were expressed as the median and interquartile range (IQR). Fisher's exact test, Wilcoxon's rank sum test, and simple linear regression models were used to compare clinical variables between RA patients and controls, as appropriate. Unadjusted associations between the RA diagnosis and QST outcomes were assessed using simple linear regression. We analyzed adjusted associations between RA and QST outcomes using multivariable linear regression, in models in which, first, RA was included and, subsequently, covariates (scores on the MOS Sleep Problems Index II, HADS depression scale, HADS anxiety scale, and Pain Catastrophizing Scale, the swollen joint count, and serum levels of CRP, TNF $\alpha$, and IL-6) were added. We also examined first-order interactions between the presence of RA and each covariate.

The strength of each association was determined using beta regression coefficients and $P$ values for the Type III sum of squares estimates, which are not affected by the order of variable entry. The beta coefficient represents the difference in outcome between RA cases and controls. The threshold for significance was set as a two-tailed $P$ value of less than or equal to 0.05 .

If the beta coefficient for RA was found to be decreased by $>20 \%$ in adjusted analyses, as compared to unadjusted analyses, we conducted mediation analyses according to the Baron and Kenny criteria (17). Specifically, we used simple and multivariable linear regression models to assess whether 1) the independent variable was significantly associated with the dependent variable, 2) the independent variable was significantly associated with the proposed mediator, and 3 ) the proposed mediator was significantly associated with the dependent variable, when adjusted for the independent variable. To confirm these results, we also assessed the proposed mediators via Sobel's test and a nonparametric bootstrapping approach, utilizing 5,000 bootstrap samples (18).

To explore the possibility that use of medications for RA may have contributed to differences in QST results between RA patients and controls, we used simple linear regression to assess the association between medications (nonsteroidal antiinflammatory drugs [NSAIDs], corticosteroids, nonbiologic disease-modifying antirheumatic drugs [DMARDs], and biologic DMARDs) and the CPM level, pressure-pain threshold, and pressure-pain tolerance level among all RA patients.

\section{RESULTS}

Characteristics of the study subjects. RA patients $(\mathrm{n}=58)$ did not differ from controls $(\mathrm{n}=54)$ in age, race, smoking status, or alcohol use (Table 1). No participants had a history of peripheral neuropathy. Six participants had diabetes; these participants were evenly distributed among the case and control groups. Eighteen patients were taking serotonin reuptake inhibitors. Five patients were taking medications for neuroleptic disor- 
Table 1. Clinical characteristics of the rheumatoid arthritis (RA) patients and healthy control subjects*

\begin{tabular}{lccr}
\hline \multicolumn{1}{c}{ Characteristic } & RA patients $(\mathrm{n}=58)$ & Controls $(\mathrm{n}=54)$ & \multicolumn{1}{c}{$P$} \\
\hline Demographic & & & \\
Age, mean \pm SD years & $60.9 \pm 9.7$ & $62.5 \pm 9.3$ & 0.34 \\
White & $53(91.4)$ & $44(81.5)$ & 0.17 \\
Current smoker & $4(6.9)$ & $4(7.4)$ & 1.00 \\
Alcohol use & $12(20.7)$ & $10(18.5)$ & 0.82 \\
Postmenopausal & $48(82.8)$ & $44(81.5)$ & 0.80 \\
Measures of inflammation/pain, median (IQR) & & & \\
Tender joint count & $3.0(1.0,5.0)$ & $0.0(0.0,0.0)$ & $<0.0001$ \\
Swollen joint count & $0.5(0.0,3.0)$ & $0.0(0.0,0.0)$ & $<0.0001$ \\
C-reactive protein, mg/liter & $1.4(0.6,4.1)$ & $0.6(0.3,1.4)$ & 0.0005 \\
IL-6, pg/ml & $2.8(1.6,6.4)$ & $1.4(1.2,2.3)$ & $<0.0001$ \\
TNF $\alpha$, pg/ml & $1.9(1.0,5.0)$ & $1.3(1.0,1.8)$ & 0.01 \\
DAS28 & $2.7(2.5,3.3)$ & $1.4(1.3,1.7)$ & $<0.0001$ \\
BPI current pain severity score & $2.0(1.0,4.0)$ & $0.0(0.0,0.0)$ & $<0.0001$ \\
Sleep/mental health measures, median (IQR) & & & \\
HADS anxiety score & $5.5(4.0,9.0)$ & $2.0(1.0,4.0)$ & $<0.0001$ \\
HADS depression score & $3.0(1.0,6.0)$ & $1.0(0.0,3.0)$ & $<0.0001$ \\
MOS Sleep Problems Index II score & $30.6(20.6,48.9)$ & $13.3(5.6,20.6)$ & $<0.0001$ \\
Pain Catastrophizing Scale score & $11.0(4.0,21.0)$ & $0.0(0.0,3.0)$ & $<0.0001$ \\
Medication use & $22(37.9)$ & $1(1.9)$ & $<0.0001$ \\
NSAIDs & $16(27.6)$ & $0(0.0)$ & $<0.0001$ \\
Corticosteroids & $38(65.5)$ & $0(0.0)$ & $<0.0001$ \\
Nonbiologic DMARDs & $27(46.6)$ & $0(0.0)$ & $<0.0001$ \\
Biologic DMARDs & & & \\
\hline
\end{tabular}

* Except where indicated otherwise, values are the number $(\%)$ of subjects. IQR = interquartile range; IL-6 $=$ interleukin-6; TNF $\alpha=$ tumor necrosis factor $\alpha$; DAS28 = Disease Activity Score in 28 joints (measured from the tender joint count, swollen joint count, and C-reactive protein level); BPI = Brief Pain Inventory; HADS = Hospital Anxiety and Depression Scale; MOS = Medical Outcomes Study; NSAIDs $=$ nonsteroidal antiinflammatory drugs; DMARDs $=$ disease-modifying antirheumatic drugs.

ders, and 1 patient was taking a tricyclic antidepressant. The percentage of subjects taking these medications did not differ significantly between RA patients and controls.

Compared to healthy control subjects, RA patients had significantly higher serum levels of CRP $(P=$ $0.0005)$, TNF $\alpha(P=0.01)$, and IL-6 $(P<0.0001)$. RA patients were also significantly more likely to have problems with anxiety, depression, sleep, and pain catastrophizing compared to controls $(P \leq 0.0001)$. RA patients were more likely to be taking NSAIDs, corticosteroids, nonbiologic DMARDs, and biologic DMARDs ( $P \leq 0.0001$ versus controls).

Clinical pain severity scores were significantly higher among RA patients as compared to controls $(P<$ 0.0001). Spearman's correlation coefficients for associations of clinical pain scores with QST measures of pain varied from -0.12 for the pressure-pain threshold at the left trapezius, to -0.33 for pressure-pain tolerance at the right wrist. The Spearman's correlation was -0.17 for the association between clinical pain intensity and the level of CPM.

Among the RA patients, the median number of swollen joints was 0.5 (IQR 0.0-3.0) (Table 1). Eight of 116 wrists $(6.9 \%)$ and 12 of 116 knees $(10.3 \%)$ were swollen. Compared to joints that were not swollen, joints that were swollen had lower pain thresholds, although this was only statistically significant for measurements at the right wrist $(P=0.05)$.

Unadjusted associations between RA and QST measures. The median CPM level was lower among RA patients (median $0.5 \mathrm{~kg} / \mathrm{cm}^{2}$, IQR $-0.1,1.6$ ) compared to control subjects without chronic pain conditions (median $1.5 \mathrm{~kg} / \mathrm{cm}^{2}$, IQR $-0.1,2.5 ; P=0.04$ ) (Figure 1 ).



Figure 1. Distribution of conditioned pain modulation levels among rheumatoid arthritis patients and healthy control subjects. 
Table 2. Measures of central pain, pressure-pain threshold, and pressure-pain tolerance in rheumatoid arthritis $(\mathrm{RA})$ patients $(\mathrm{n}=58)$ compared to healthy controls $(\mathrm{n}=54)^{*}$

\begin{tabular}{|c|c|c|c|c|c|c|c|c|}
\hline \multirow[b]{2}{*}{ QST outcome } & \multicolumn{2}{|c|}{ Median (IQR) $\mathrm{kg} / \mathrm{cm}^{2}$} & \multicolumn{2}{|c|}{ Model $1 \dagger$} & \multicolumn{2}{|c|}{ Model $2 \dagger$} & \multicolumn{2}{|c|}{ Model $3 \dagger$} \\
\hline & RA patients & Controls & $\beta$ & $P$ & $\beta$ & $P$ & $\beta$ & $P$ \\
\hline Central pain, CPM & $0.5(-0.1,1.6)$ & $1.5(-0.1,2.5)$ & -0.59 & 0.04 & $-0.19 \ddagger$ & 0.57 & -0.60 & 0.09 \\
\hline \multicolumn{9}{|l|}{ Pressure-pain threshold } \\
\hline \multicolumn{9}{|l|}{ Wrist } \\
\hline Left & $6.1(4.8,8.5)$ & $7.7(5.7,11.0)$ & -0.99 & 0.05 & -1.21 & 0.04 & $-0.40 \ddagger$ & 0.49 \\
\hline Right & $6.8(4.8,8.8)$ & $8.1(6.0,10.3)$ & -1.06 & 0.03 & -0.99 & 0.08 & $-0.54 \ddagger$ & 0.32 \\
\hline \multicolumn{9}{|l|}{ Knee } \\
\hline Left & $8.4(6.0,11.0)$ & $9.2(7.5,11.0)$ & -0.76 & 0.10 & -0.90 & 0.10 & $-0.05 \ddagger$ & 0.93 \\
\hline Right & $7.5(5.9,11.0)$ & $9.4(7.3,11.0)$ & -1.17 & 0.01 & -1.30 & 0.02 & $-0.67 \ddagger$ & 0.19 \\
\hline \multicolumn{9}{|l|}{ Thumb } \\
\hline Left & $6.4(4.4,8.3)$ & $6.9(5.7,11.0)$ & -0.57 & 0.25 & - & - & - & - \\
\hline Right & $6.7(5.0,9.8)$ & $8.1(5.7,9.8)$ & -0.53 & 0.31 & - & - & - & - \\
\hline \multicolumn{9}{|l|}{ Trapezius } \\
\hline Left & $5.5(3.8,7.8)$ & $6.2(4.1,8.1)$ & -0.37 & 0.45 & - & - & - & - \\
\hline Right & $5.2(3.8,7.9)$ & $6.4(4.2,9.3)$ & -0.61 & 0.23 & - & - & - & - \\
\hline \multicolumn{9}{|l|}{ Pressure-pain tolerance } \\
\hline \multicolumn{9}{|l|}{ Wrist } \\
\hline Left & $8.0(6.2,11.0)$ & $10.4(7.3,11.0)$ & -1.04 & 0.02 & -1.23 & 0.01 & $-0.79 \ddagger$ & 0.11 \\
\hline Right & $8.8(6.3,11.0)$ & $11.0(8.1,11.0)$ & -1.28 & 0.002 & -1.31 & 0.007 & $-0.89 \ddagger$ & 0.05 \\
\hline \multicolumn{9}{|l|}{ Knee } \\
\hline Left & $9.8(7.8,11.0)$ & $11.0(8.9,11.0)$ & -0.90 & 0.03 & -0.89 & 0.06 & $-0.40 \ddagger$ & 0.35 \\
\hline Right & $9.0(7.4,11.0)$ & $11.0(9.8,11.0)$ & -1.28 & 0.001 & -1.39 & 0.004 & $-0.86 \ddagger$ & 0.20 \\
\hline \multicolumn{9}{|l|}{ Thumb } \\
\hline Left & $8.5(6.2,11.0)$ & $9.0(7.1,11.0)$ & -0.55 & 0.23 & - & - & - & - \\
\hline Right & $9.8(7.2,11.0)$ & $11.0(8.5,11.0)$ & -0.60 & 0.17 & - & - & - & - \\
\hline \multicolumn{9}{|l|}{ Trapezius } \\
\hline Left & $7.1(4.8,9.5)$ & $8.2(5.2,10.5)$ & -0.51 & 0.32 & - & - & - & - \\
\hline Right & $7.0(4.8,9.0)$ & $8.8(5.2,11.0)$ & -0.82 & 0.12 & - & - & - & - \\
\hline
\end{tabular}

* QST = quantitative sensory testing; IQR = interquartile range; CPM = conditioned pain modulation.

$\dagger$ Values are the beta coefficient and $P$ value for the association between RA and each outcome, as assessed in model 1 (unadjusted), model 2 (adjusted for sleep problems), and model 3 (adjusted for pain catastrophizing score).

$\ddagger$ The beta coefficient is decreased $\geq 20 \%$ from the unadjusted value in model 1 . Because mediation can occur only when the original association between the independent and dependent variable is significant, the beta coefficients and $P$ values are provided only for models in which the $P$ value for the original association between RA and the outcome was $\leq 0.10$.

When compared to controls, RA patients had significantly lower pressure-pain thresholds at the wrists and the right knee $(P \leq 0.05)$, and had lower pressure-pain tolerance $(P \leq 0.03)$ at the wrists and knees bilaterally (Table 2). The pain thresholds and pain tolerance levels at the thumbs and trapezius muscles were also lower among RA patients compared to controls, but these differences were not statistically significant (Table 2).

Adjusted associations between RA and CPM. The beta coefficient for the association between RA and level of CPM decreased by $>20 \%$ when the MOS sleep problems score was added to linear regression models assessing the association between RA and CPM, suggesting that sleep problems may either mediate or confound this association (Table 2). In analyses stratified by clinical pain intensity scores, similar decrements in the beta coefficient for the association between RA and level of CPM were noted.

No significant decreases in the beta coefficients for RA were noted when the pain catastrophizing score,
HADS anxiety score, HADS depression score, swollen joint count, serum CRP level, serum TNF $\alpha$ level, or serum IL-6 level was added individually as a covariate to the models. No significant changes in the beta coefficients for RA were noted when diabetes mellitus, use of tricyclic antidepressant drugs, use of serotonin reuptake inhibitors, or use of medications for neuroleptic disorders was added individually to the models.

Adjusted associations between RA and pain threshold and pain tolerance. The beta coefficients for the association between RA and pain thresholds and pain tolerance levels at the wrists and knees decreased by $>20 \%$ when the pain catastrophizing score was added to the models, suggesting that the extent of pain catastrophizing may either mediate or confound these relationships (Table 2). The beta coefficient for the association between RA and the pressure-pain threshold at the left wrist increased by $>20 \%$ when the MOS sleep problems score was added to the model (Table 2), and the beta coefficient for the association between RA and 
Table 3. Analyses of sleep disturbances as a mediator of the association between rheumatoid arthritis (RA) and each quantitative sensory testing (QST) outcome, according to the Baron and Kenny criteria, Sobel's test, and bootstrapping analyses*

\begin{tabular}{|c|c|c|c|c|c|}
\hline \multirow[b]{2}{*}{ QST outcome } & \multicolumn{3}{|c|}{ Baron and Kenny criteria } & \multirow[b]{2}{*}{$\begin{array}{c}\text { Sobel's } \\
\text { test }\end{array}$} & \multirow[b]{2}{*}{$\begin{array}{c}\text { Bootstrapping analysis } \\
\text { of the indirect effect } \\
\text { of RA on QST } \\
\text { outcome, through } \\
\text { sleep problems }\end{array}$} \\
\hline & $\begin{array}{c}\text { Association } \\
\text { between RA } \\
\text { and QST } \\
\text { outcome }\end{array}$ & $\begin{array}{c}\text { Association } \\
\text { between RA } \\
\text { and sleep } \\
\text { problems }\end{array}$ & $\begin{array}{c}\text { Association between } \\
\text { sleep problems and } \\
\text { QST outcome, } \\
\text { adjusted for RA }\end{array}$ & & \\
\hline Central pain, CPM & 0.04 & $<0.0001$ & 0.03 & 0.04 & $-0.74,-0.04$ \\
\hline \multicolumn{6}{|l|}{ Pressure-pain threshold } \\
\hline \multicolumn{6}{|l|}{ Wrist } \\
\hline Left & 0.05 & $<0.0001$ & 0.49 & 0.49 & $-0.76,1.22$ \\
\hline Right & 0.03 & $<0.0001$ & 0.83 & 0.83 & $-1.13,0.83$ \\
\hline \multicolumn{6}{|l|}{ Knee } \\
\hline Left & 0.10 & $<0.0001$ & 0.63 & 0.63 & $-0.67,0.92$ \\
\hline Right & 0.01 & $<0.0001$ & 0.65 & 0.66 & $-0.72,1.02$ \\
\hline \multicolumn{6}{|l|}{ Pressure-pain tolerance } \\
\hline \multicolumn{6}{|l|}{ Wrist } \\
\hline Left & 0.02 & $<0.0001$ & 0.41 & 0.42 & $-0.58,1.04$ \\
\hline Right & 0.002 & $<0.0001$ & 0.89 & 0.89 & $-0.79,0.77$ \\
\hline \multicolumn{6}{|l|}{ Knee } \\
\hline Left & 0.03 & $<0.0001$ & 0.98 & 0.98 & $-0.78,0.65$ \\
\hline Right & 0.001 & $<0.0001$ & 0.67 & 0.67 & $-0.64,0.88$ \\
\hline
\end{tabular}

* Each row represents a separate model. For associations tested according to the Baron and Kenny criteria and Sobel's test, the values are $P$ values, while values in bootstrapping analyses are the $95 \%$ confidence interval of the indirect effect. $\mathrm{CPM}=$ conditioned pain modulation.

the pressure-pain threshold at the left knee increased by $>20 \%$ when the HADS anxiety score was added to the model. No changes in the beta coefficients for RA were noted when the HADS depression score, swollen joint count, serum CRP level, serum TNF $\alpha$ level, or serum IL-6 level was added individually to the models assessing the association between RA and pain threshold and pain tolerance at the wrists and knees.

Associations in mediation analyses. Mediation analyses were conducted to identify mediators of the association between RA and CPM levels, and between RA and pressure-pain thresholds and pressure-pain tolerance levels at the wrists and knees. All 3 Baron and Kenny criteria were satisfied when the MOS sleep problems score was assessed as a mediator of the association between RA and the level of CPM: 1) RA was significantly associated with the level of CPM $(P=$ $0.04)$; 2) RA was significantly associated with the MOS Sleep Problems Index II score $(\mathrm{P}<0.0001)$; and 3$)$ the MOS Sleep Problems Index II score was significantly associated with the level of CPM after adjustment for the presence of RA $(P=0.03)$ (Table 3 and Figures 2A and $\mathrm{B})$. According to the results from Sobel's test, the association between RA and the level of CPM was significantly reduced by inclusion of the MOS Sleep Problems Index II score in the model $(\beta=-0.59$ in unadjusted analyses versus $\beta=-0.19$ after adjustment for sleep problems; $P=0.04$ ) (Figures 2A and B). Moreover, based on analyses utilizing the bootstrapping approach, the $95 \%$ confidence interval for the indirect effect of RA on the CPM level, mediated by sleep problems, did not overlap with zero (Table 3).

A

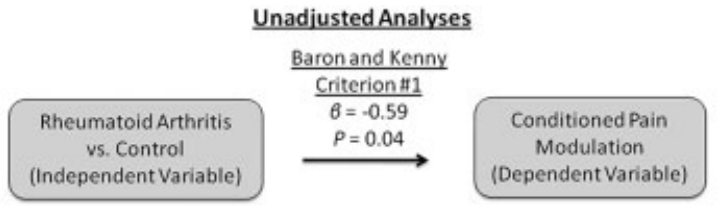

B
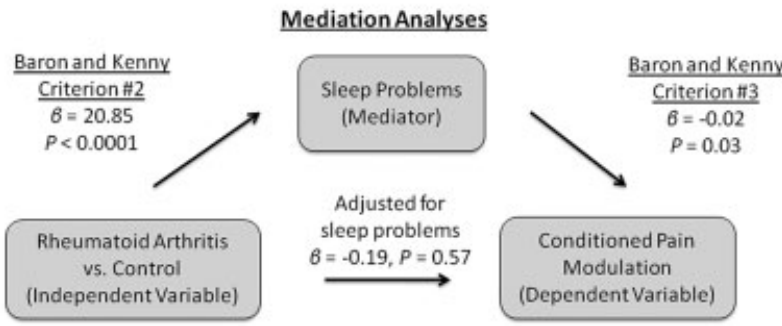

Figure 2. A, Unadjusted association between rheumatoid arthritis and conditioned pain modulation (Baron and Kenny criterion \#1). B, Mediation analyses showing 1) the association between rheumatoid arthritis and conditioned pain modulation, adjusted for sleep problems, and 2) the associations between rheumatoid arthritis and sleep problems (Baron and Kenny criterion \#2) and sleep problems and conditioned pain modulation, adjusted for rheumatoid arthritis (Baron and Kenny criterion \#3). 
The Baron and Kenny criteria were not satisfied when pain catastrophizing scores were analyzed as a mediator of the relationship between RA and pressurepain thresholds and pressure-pain tolerance levels at the wrists and knees. Although we found that RA was significantly associated with the pain threshold and the pain tolerance level, and that RA was significantly associated with the pain catastrophizing score, no significant association between the pain catastrophizing score and either the pain threshold or pain tolerance level was seen after adjustment for the presence of RA. Furthermore, neither the results of Sobel's test nor findings from the bootstrapping approach indicated that pain catastrophizing was a mediator of the association between RA and pain threshold and pain tolerance at the wrists and knees.

Interactions. Analyses to assess the effects of interaction between RA diagnosis and the MOS Sleep Problems Index II score and between RA diagnosis and the pain catastrophizing score on QST outcomes showed that neither of these interactions was significantly associated with the level of CPM. However, the interaction between RA diagnosis and the MOS Sleep Problems Index II score was significantly associated with the pressure-pain threshold at the left thumb $(P=0.03)$, and the interaction between RA diagnosis and the pain catastrophizing score was significantly associated with the pressure-pain threshold at the right knee $(P=0.04)$.

Associations with medication use. Among RA patients, use of nonbiologic DMARDs was significantly associated with higher pain thresholds $(P \leq 0.05)$ at the left thumb and right trapezius, and also significantly associated with higher pain tolerance $(P \leq 0.05)$ at the thumbs and trapezius muscles bilaterally. Use of nonbiologic DMARDs was not significantly associated with the level of CPM or the pain threshold and pain tolerance level at the wrists and knees. Use of NSAIDs, corticosteroids, and biologic DMARDs was not associated with the level of CPM or the pain threshold and pain tolerance level at any site.

\section{DISCUSSION}

In this study, we examined QST measures of central pain among RA patients compared to healthy controls. Our results showed that RA patients have attenuated CPM compared to controls, suggesting that descending analgesic mechanisms are impaired. This observation is consistent with studies comparing the level of CPM between patients in other chronic pain populations (e.g., fibromyalgia and osteoarthritis) and healthy controls $(19,20)$. Functional neuroimaging studies have also identified changes in neural activity in the periaqueductal grey, anterior cingulate cortex, and other pain-responsive areas of the brain in response to experimental induction of CPM $(21,22)$. Taken together, these studies suggest that a set of common central mechanisms, including impaired CPM, may contribute to numerous chronic pain states. The association between these measures and clinical pain intensity, however, has varied, with some studies showing a significant inverse correlation between the CPM level and clinical pain measures (23), and other studies showing no association (24).

Only one other study has examined the role of CPM in RA. In contrast to our study, Leffler et al reported no statistically significant differences in the level of CPM between 21 RA patients and 21 healthy controls (4). The differences in results may reflect differences in statistical power, as the present study had over twice as many subjects. Similar to our study, the mean level of CPM in the Leffler et al study was lower (but not significantly $[P<0.05]$ ) among RA patients compared to pain-free controls.

Pressure-pain thresholds and pressure-pain tolerances were also lower among RA patients than among controls in our study. However, in contrast to previous studies $(4,5)$, these differences were only significant in the joints, but not at sites outside the joints. The stronger association with pain threshold and pain tolerance in the joints may be due, in part, to peripheral sensitization resulting from inflammation of the joints. In animal models, it is well established that peripheral inflammation can sensitize peripheral nerve endings, leading to lower pain thresholds (25). In a previous study, we also demonstrated that the serum CRP level was significantly associated with the pain threshold at the wrist, but not at sites outside the joints (26). Taken together, these results provide further support for the idea that inflammation is related to peripheral sensitization in patients with RA.

Interaction analyses did not suggest a differential association of either sleep problems or pain catastrophizing with the level of CPM, depending on the presence of RA. Although the $P$ values for the association between 1) RA diagnosis by MOS Sleep Problems Index II score and pressure-pain threshold at the left thumb, and 2) RA diagnosis by pain catastrophizing score and pressure-pain threshold at the right knee were both less than 0.05 , the clinical significance of these associations is unclear, since these interactions were analyzed ad hoc, and interactions at other sites were not significant. 
Statistical significance may have been a by-product of multiple comparisons. Future analyses are needed to replicate these results.

The association between RA and CPM level was greatly attenuated when sleep problems were included as a covariate in the multivariable model. This observation suggests that sleep problems may confound or mediate the relationship between RA and CPM. Results of mediation analyses, including fulfillment of the Baron and Kenny criteria as well as results from Sobel's test and the confidence intervals obtained from the nonparametric bootstrapping approach, all indicated that the indirect effect of RA on the level of CPM through sleep problems (i.e., the effect that occurred due to sleep problems, rather than the direct effect of RA itself on the CPM) was statistically significant.

In the proposed mediation pathway (Figure 2B), RA is associated with a lower CPM level because 1) RA patients have more sleep problems, and 2) sleep problems lead to impaired CPM. Sleep disturbances are more common among RA patients than among the general population (27), occurring in $50-70 \%$ of RA patients (28). Although no studies have examined the association between sleep fragmentation and CPM levels among RA patients, studies of patients with temporomandibular joint disorder suggest that sleep disturbances are cross-sectionally associated with experimental pain sensitivity (29) and prospectively associated with clinical pain severity (30). In addition, studies of healthy adults and adults with temporomandibular joint disorder indicate that sleep fragmentation is significantly associated with decreases in the CPM level $(24,31)$.

The relationship between sleep problems and pain, however, is complex. While sleep problems appear to mediate the association between RA and the level of CPM, other variables, such as pain itself, may factor into this relationship. Given that control subjects were excluded if they reported the presence of chronic pain, cases and controls differed in the pain experience. A study of 106 RA patients showed that pain significantly contributes to sleep problems (32). Thus, the appearance of mediation by sleep may actually be the result of mediation by chronic pain, as both may be on the same causal pathway. However, because pain was an exclusion criterion for the controls, it was not possible to assess pain as a confounder or mediator in these analyses. Analyses stratified by clinical pain intensity in the RA group did not suggest differences in the mediating effect of sleep problems on the CPM level.

While sleep problems altered the strength of association between RA and CPM, pain catastrophizing diminished the association between RA and pain threshold and pain tolerance at the wrists and knees. However, mediation analyses did not support the role of pain catastrophizing as a mediator of the relationship between RA and pain threshold or pain tolerance. The associations between pain catastrophizing and pain threshold and pain tolerance were not significant after adjusting for the presence of RA, suggesting that pain catastrophizing may be collinear with other factors associated with the RA diagnosis. Alternatively, the lack of significance in these tests may be due to the small sample size, limited distribution of pain catastrophizing scores, and/or asymmetric distributions of the indirect effect of RA on pain threshold and pain tolerance.

Although no studies have examined the association between the extent of pain catastrophizing and either the CPM level or pain threshold and pain tolerance levels in RA, a greater pain catastrophizing score has been associated with high self-reported pain severity among RA patients $(33,34)$. Pain catastrophizing scores are also inversely associated with pressure-pain thresholds and pressure-pain tolerance levels in patients with fibromyalgia and patients with osteoarthritis (35-38). Future studies are needed to elucidate the role of pain catastrophizing in the processing and ultimate expression of pain in RA.

This study has several limitations. First, the crosssectional design makes it impossible to assess causality. Because the distinction between confounding and mediation is based on a presumed causal relationship among variables, this study cannot distinguish between confounding and mediation, even though the results of mediation analyses were statistically significant $(39,40)$. In studies of other populations (e.g., patients after knee arthroscopy), a causal relationship between sleep problems and pain has been suggested based on improvements in pain after treatment of sleep disturbances $(41,42)$. In future studies, it will be important to assess whether interventions that improve sleep in RA patients could "normalize" CPM measures. Larger, longitudinal studies examining the interplay between pain, mental health, sleep problems, CPM, pain threshold, and pain tolerance are also needed to understand how these factors interact in shaping CNS processing of painrelated information in RA.

Other potential limitations include confounding by medications. Although we excluded individuals taking opioids, participants could continue to take other medications that may alter the CPM level, pain threshold, and pain tolerance level. Use of NSAIDs, cortico- 
steroids, and DMARDs was observed almost exclusively in RA patients, and thus may be the cause of differences in the CPM level, pain threshold, and pain tolerance between RA patients and controls. However, among RA patients, none of these medications, except nonbiologic DMARDs, was significantly associated with the CPM level, pain threshold, or pain tolerance. Nonbiologic DMARDs were associated with a high pressure-pain threshold and high pressure-pain tolerance level at the thumb and trapezius, but these associations were in the opposite direction of what would be expected if nonbiologic DMARDs were a proxy for RA diagnosis. Future studies are needed to examine the effect of medications on central pain mechanisms.

Finally, it is possible that the use of large cotton gloves to blind the assessor to RA/control status altered the pain-testing results. In a pilot study of 8 healthy women, the median difference in pain threshold between participants wearing gloves and those not wearing gloves was $0.30 \mathrm{~kg} / \mathrm{cm}^{2}$, indicating that gloves do not significantly alter pain thresholds in healthy controls. In a separate study of 8 women with RA, the median difference in pain threshold was $0.74 \mathrm{~kg} / \mathrm{cm}^{2}$. These results suggest that, if anything, gloves increase pain thresholds in RA patients compared to controls, biasing results toward the null. Thus, the use of gloves, which was necessary for blinding, may have decreased our power, but there was no evidence that this confounded the analysis of group differences in CPM (which did not involve a gloved site) or produced spurious findings.

In conclusion, RA patients had lower CPM levels and lower pressure-pain threshold and pressure-pain tolerance at the joints when compared to pain-free controls. The association between RA and diminished CPM may be mediated by sleep problems. These results highlight the importance of considering central pain mechanisms when evaluating and addressing pain in chronic inflammatory diseases such as RA. Future studies are needed to examine whether improving sleep and managing pain catastrophizing may prevent or reverse the development of abnormal pain mechanisms and, ultimately, improve pain control in RA. Functional neuroimaging studies may also be illuminating, as several recent functional magnetic resonance imaging studies $(22,43)$ have investigated the neural underpinnings of CPM.

\section{AUTHOR CONTRIBUTIONS}

All authors were involved in drafting the article or revising it critically for important intellectual content, and all authors approved the final version to be published. Dr. Lee had full access to all of the data in the study and takes responsibility for the integrity of the data and the accuracy of the data analysis.

Study conception and design. Lee, Lu, Edwards, Wasan, Clauw, Karlson.

Acquisition of data. Lee, Nassikas.

Analysis and interpretation of data. Lee, Lu, Edwards, Wasan, Clauw, Solomon, Karlson.

\section{REFERENCES}

1. Lee YC, Cui J, Lu B, Frits ML, Iannaccone CK, Shadick NA, et al. Pain persists in DAS28 rheumatoid arthritis remission but not in ACR/EULAR remission: a longitudinal observational study. Arthritis Res Ther 2011;13:R83.

2. Huskisson EC, Hart FD. Pain threshold and arthritis. Br Med J 1972;4:193-5.

3. Gerecz-Simon EM, Tunks ER, Heale JA, Kean WF, Buchanan WW. Measurement of pain threshold in patients with rheumatoid arthritis, osteoarthritis, ankylosing spondylitis, and healthy controls. Clin Rheumatol 1989;8:467-74.

4. Leffler AS, Kosek E, Lerndal T, Nordmark B, Hansson P. Somatosensory perception and function of diffuse noxious inhibitory controls (DNIC) in patients suffering from rheumatoid arthritis. Eur J Pain 2002;6:161-76.

5. Edwards RR, Wasan AD, Bingham CO III, Bathon J, Haythornthwaite JA, Smith MT, et al. Enhanced reactivity to pain in patients with rheumatoid arthritis. Arthritis Res Ther 2009;11: R61.

6. Millan MJ. Descending control of pain. Prog Neurobiol 2002;66: 355-474.

7. Lee YC, Nassikas NJ, Clauw DJ. The role of the central nervous system in the generation and maintenance of chronic pain in rheumatoid arthritis, osteoarthritis and fibromyalgia. Arthritis Res Ther 2011;13:211.

8. Van Wijk G, Veldhuijzen DS. Perspective on diffuse noxious inhibitory controls as a model of endogenous pain modulation in clinical pain syndromes. J Pain 2010;11:408-19.

9. Prevoo ML, van 't Hof MA, Kuper HH, van Leeuwen MA, van de Putte LB, van Riel PL. Modified disease activity scores that include twenty-eight-joint counts: development and validation in a prospective longitudinal study of patients with rheumatoid arthritis. Arthritis Rheum 1995;38:44-8.

10. Bjelland I, Dahl AA, Haug TT, Neckelmann D. The validity of the Hospital Anxiety and Depression Scale: an updated literature review. J Psychosom Res 2002;52:69-77.

11. Hays RD, Stewart AL. Sleep measures. In: Stewart AL, Ware JE $\mathrm{Jr}$, editors. Measuring functioning and well-being: the Medical Outcomes Study approach. Durham, NC: Durham University Press; 1992. p. 235-59.

12. Sullivan MJ, Bishop SR, Pivik J. The Pain Catastrophizing Scale: development and validation. Psychol Assess 1995;7:524-32.

13. Lee YC, Chibnik LB, Fossel AH, Solomon DH, Clauw DJ, Karlson EW. The reproducibility of pressure pain thresholds dolorimetry measurements in rheumatoid arthritis patients [abstract]. Arthritis Rheum 2008;58 Suppl:S283-4.

14. Wilder-Smith OH, Schreyer T, Scheffer GJ, Arendt-Nielsen L. Patients with chronic pain after abdominal surgery show less preoperative endogenous pain inhibition and more postoperative hyperalgesia: a pilot study. J Pain Palliat Care Pharmacother 2010;24:119-28.

15. Goodin BR, McGuire L, Allshouse M, Stapleton L, Haythornthwaite JA, Burns N, et al. Associations between catastrophizing and endogenous pain-inhibitory processes: sex differences. J Pain 2009;10:180-90.

16. Lariviere M, Goffaux P, Marchand S, Julien N. Changes in pain 
perception and descending inhibitory controls start at middle age in healthy adults. Clin J Pain 2007;23:506-10.

17. Baron RM, Kenny DA. The moderator-mediator variable distinction in social psychological research: conceptual, strategic, and statistical considerations. J Pers Soc Psychol 1986;51:1173-82.

18. Preacher KJ, Hayes AF. SPSS and SAS procedures for estimating indirect effects in simple mediation models. Behav Res Methods Instrum Comput 2004;36:717-31.

19. Normand E, Potvin S, Gaumond I, Cloutier G, Corbin JF, Marchand S. Pain inhibition is deficient in chronic widespread pain but normal in major depressive disorder. J Clin Psychiatry 2011;72:219-24.

20. Arendt-Nielsen L, Nie H, Laursen MB, Laursen BS, Madeleine P, Simonsen $\mathrm{OH}$, et al. Sensitization in patients with painful knee osteoarthritis. Pain 2010;149:573-81.

21. Derbyshire SW, Osborn J. Offset analgesia is mediated by activation in the region of the periaqueductal grey and rostral ventromedial medulla. Neuroimage 2009;47:1002-6.

22. Sprenger C, Bingel U, Buchel C. Treating pain with pain: supraspinal mechanisms of endogenous analgesia elicited by heterotopic noxious conditioning stimulation. Pain 2011;152:428-39.

23. Edwards RR, Ness TJ, Weigent DA, Fillingim RB. Individual differences in diffuse noxious inhibitory controls (DNIC): association with clinical variables. Pain 2003;106:427-37.

24. Edwards RR, Grace E, Peterson S, Klick B, Haythornthwaite JA, Smith MT. Sleep continuity and architecture: associations with pain-inhibitory processes in patients with temporomandibular joint disorder. Eur J Pain 2009;13:1043-7.

25. Schaible HG, Ebersberger A, Von Banchet GS. Mechanisms of pain in arthritis. Ann N Y Acad Sci 2002;966:343-54.

26. Lee YC, Chibnik LB, Lu B, Wasan AD, Edwards RR, Fossel AH, et al. The relationship between disease activity, sleep, psychiatric distress and pain sensitivity in rheumatoid arthritis: a crosssectional study. Arthritis Res Ther 2009;11:R160.

27. Clegg DO, Reda DJ, Harris CL, Klein MA, O'Dell JR, Hooper $\mathrm{MM}$, et al. Glucosamine, chondroitin sulfate, and the two in combination for painful knee osteoarthritis. N Engl J Med 2006; 354:795-808.

28. Abad VC, Sarinas PS, Guilleminault C. Sleep and rheumatologic disorders. Sleep Med Rev 2008;12:211-28.

29. Smith MT, Wickwire EM, Grace EG, Edwards RR, Buenaver LF, Peterson S, et al. Sleep disorders and their association with laboratory pain sensitivity in temporomandibular joint disorder. Sleep 2009;32:779-90.

30. Quartana PJ, Wickwire EM, Klick B, Grace E, Smith MT Naturalistic changes in insomnia symptoms and pain in temporomandibular joint disorder: a cross-lagged panel analysis. Pain 2010;149:325-31.
31. Smith MT, Edwards RR, McCann UD, Haythornthwaite JA. The effects of sleep deprivation on pain inhibition and spontaneous pain in women. Sleep 2007;30:494-505.

32. Nicassio PM, Ormseth SR, Kay M, Custodio M, Irwin MR, Olmstead R, et al. The contribution of pain and depression to self-reported sleep disturbance in patients with rheumatoid arthritis. Pain 2012;153:107-12.

33. Covic T, Adamson B, Spencer D, Howe G. A biopsychosocial model of pain and depression in rheumatoid arthritis: a 12-month longitudinal study. Rheumatology (Oxford) 2003;42:1287-94.

34. Edwards RR, Giles J, Bingham CO III, Campbell C, Haythornthwaite JA, Bathon J. Moderators of the negative effects of catastrophizing in arthritis. Pain Med 2010;11:591-9.

35. Edwards RR, Cahalan C, Mensing G, Smith M, Haythornthwaite JA. Pain, catastrophizing, and depression in the rheumatic diseases. Nat Rev Rheumatol 2011;7:216-24.

36. Geisser ME, Casey KL, Brucksch CB, Ribbens CM, Appleton BB, Crofford LJ. Perception of noxious and innocuous heat stimulation among healthy women and women with fibromyalgia: association with mood, somatic focus, and catastrophizing. Pain 2003; 102:243-50.

37. France CR, Keefe FJ, Emery CF, Affleck G, France JL, Waters S, et al. Laboratory pain perception and clinical pain in postmenopausal women and age-matched men with osteoarthritis: relationship to pain coping and hormonal status. Pain 2004;112 274-81.

38. Campbell CM, Kronfli T, Buenaver LF, Smith MT, Berna C, Haythornthwaite JA, et al. Situational versus dispositional measurement of catastrophizing: associations with pain responses in multiple samples. J Pain 2010;11:443-53.e2.

39. MacKinnon DP, Krull JL, Lockwood CM. Equivalence of the mediation, confounding and suppression effect. Prev Sci 2000;1: $173-81$.

40. Babyak MA. Understanding confounding and mediation. Evid Based Ment Health 2009;12:68-71.

41. Tashjian RZ, Banerjee R, Bradley MP, Alford W, Fadale PD. Zolpidem reduces postoperative pain, fatigue, and narcotic consumption following knee arthroscopy: a prospective randomized placebo-controlled double-blinded study. J Knee Surg 2006;19: $105-11$.

42. Tompkins M, Plante M, Monchik K, Fleming B, Fadale P. The use of a non-benzodiazepine hypnotic sleep-aid (Zolpidem) in patients undergoing ACL reconstruction: a randomized controlled clinical trial. Knee Surg Sports Traumatol Arthrosc 2011;19:787-91.

43. Piche M, Arsenault M, Rainville P. Cerebral and cerebrospinal processes underlying counterirritation analgesia. J Neurosci 2009; 29:14236-46. 